\title{
Susceptibility loci for murine HIV-associated nephropathy encode trans-regulators of podocyte gene expression
}

\author{
Natalia Papeta, ${ }^{1}$ Ka-Tak Chan, ${ }^{1}$ Sindhuri Prakash, ${ }^{1}$ Jeremiah Martino, ${ }^{1}$ Krzysztof Kiryluk, ${ }^{1}$ \\ David Ballard, ${ }^{2}$ Leslie A. Bruggeman, ${ }^{3}$ Rachelle Frankel, ${ }^{1}$ Zongyu Zheng, ${ }^{1}$ Paul E. Klotman, ${ }^{4}$ \\ Hongyu Zhao, ${ }^{2}$ Vivette D. D’Agati, ${ }^{5}$ Richard P. Lifton, ${ }^{2,6}$ and Ali G. Gharavi ${ }^{1}$

\begin{abstract}
1Department of Medicine, Columbia University College of Physicians and Surgeons, New York, New York, USA. ${ }^{2}$ Department of Genetics, Yale University School of Medicine, New Haven, Connecticut, USA. ${ }^{3}$ Department of Medicine, MetroHealth Medical Center, Case Western Reserve University School of Medicine, Cleveland, Ohio, USA. ${ }^{4}$ Department of Medicine, Mount Sinai School of Medicine, New York, New York, USA. ${ }^{5}$ Department of Pathology, Columbia University College of Physicians and Surgeons, New York, New York, USA.
\end{abstract} \\ ${ }^{6}$ Howard Hughes Medical Institute, Yale University School of Medicine, New Haven, Connecticut, USA.
}

\begin{abstract}
Multiple studies have linked podocyte gene variants to diverse sporadic nephropathies, including HIV-1-associated nephropathy (HIVAN). We previously used linkage analysis to identify a major HIVAN susceptibility locus in mouse, HIVAN1. We performed expression quantitative trait locus (eQTL) analysis of podocyte genes in HIV-1 transgenic mice to gain further insight into genetic susceptibility to HIVAN. In 2 independent crosses, we found that transcript levels of the podocyte gene nephrosis 2 homolog $(N p h s 2)$, were heritable and controlled by an ancestral cis-eQTL that conferred a 3-fold variation in expression and produced reactive changes in other podocyte genes. In addition, $N p h s 2$ expression was controlled by 2 trans-eQTLs that localized to the nephropathy susceptibility intervals HIVAN1 and HIVAN2. Transregulation of podocyte genes was observed in the absence of HIV-1 or glomerulosclerosis, indicating that nephropathy susceptibility alleles induce latent perturbations in the podocyte expression network. Presence of the HIV-1 transgene interfered with transregulation, demonstrating effects of gene-environment interactions on disease. These data demonstrate that transcript levels of $N p h s 2$ and related podocyte-expressed genes are networked and suggest that the genetic lesions introduced by HIVAN susceptibility alleles perturb this regulatory pathway and transcriptional responses to HIV-1, increasing susceptibility to nephropathy.
\end{abstract}

\section{Introduction}

In the past decade, studies in humans and murine models have implicated defects in the glomerular visceral epithelial cells, the podocytes, as a principal factor in the development of nephropathy (1-7). Podocytes are highly differentiated epithelial cells that establish and maintain the glomerular basement membrane and the slit diaphragm. Inherited defects in genes that are specific to or highly expressed in podocytes result in Mendelian forms of nephropathy (e.g., nephrosis 1 homolog [NPHS1], NPHS2, $A C T N-4, C D 2$-associated protein [CD2AP], phospholipase C, $\varepsilon 1$ [PLCE1], TRPC6; refs. 1-6). Most recently, 2 studies have also reported strong association of noncoding variants in the podocyte-expressed gene myosin, heavy chain 9, non-muscle (MYH9) with susceptibility to primary focal segmental glomerulosclerosis, HIV-1-associated nephropathy (HIVAN), and nondiabetic endstage renal failure among African Americans $(8,9)$. These data explain the striking familial aggregation of these pathologically distinct nephropathies among African-Americans $(10,11)$ and confirm that podocyte defects are a major risk factor for multiple common forms of kidney failure. Interestingly, coding mutations

Conflict of interest: The authors have declared that no conflict of interest exists. Nonstandard abbreviations used: B6, C57BL/6J; BC, backcross; BUN, blood urea nitrogen; CAST, CAST/Eij; Cd2ap, CD2-associated protein; eQTL, expression QTL; FVB, FVB/NJ; HIVAN, HIV-1-associated nephropathy; Kirrel, kin of IRRE like; Myb9, myosin, heavy chain 9, non-muscle; Nphs1, nephrosis 1 homolog; Plce1, phospholipase C, $\varepsilon 1$; QTL, quantitative trait locus; Synpo, synaptopodin; Wt1, Wilms tumor 1 homolog. Citation for this article: J. Clin. Invest. 119:1178-1188 (2009). doi:10.1172/JCI37131. in $\mathrm{MYH9}$ cause overlapping dominant syndromic disorders predominantly characterized by macrothrombocytopenia and deafness but can also produce proteinuric nephropathy (May-Hegglin anomaly and Fechtner, Epstein, and Sebastian syndromes; refs. 12-16). The mechanism by which coding or noncoding MYH9 variants result in diverse nephropathies is not clear.

Several studies have suggested a requirement for gene-gene and gene-environment interaction for determination of complex nephropathy phenotypes $(4,17-20)$. For example, $C d 2 a p$ haploinsufficient mice do not develop overt nephropathy but have increased susceptibility to experimental glomerular injury or develop nephropathy in conjunction with a null allele in either the synaptopodin (Synpo) gene or the Fyn proto-oncogene $(4,21)$. These data suggest that podocytes can counteract moderate genetic lesions and restore cellular homeostasis without organ failure, but additional insults produce decompensation and disease. From this perspective, genetic susceptibility represents a compensated state that is unmasked upon exposure to additional genetic or environmental insults. However, molecular evidence of a regulated compensatory pathway has not been clearly documented, with genetic interactions being attributed to disruption of physical interaction between encoded gene products (17). Altered expression profiles of podocyte genes have also been demonstrated in humans and animals with genetic or acquired nephropathy $(19,22,23)$, but in these settings, primary adaptive changes cannot be differentiated from secondary effects induced by glomerulosclerosis. Identification of such transcriptional networks and their co-regulated com- 
Table 1

Significant and suggestive loci for nephropathy-related traits in the TgFVB $\times$ B6 F2 intercross

\begin{tabular}{lcccc} 
Trait & Chromosome & $\begin{array}{c}\text { Location } \\
\text { (lod -2), in Mb }\end{array}$ & $\begin{array}{c}\text { lod } \\
\text { score }\end{array}$ & $\begin{array}{c}\text { Allele associated } \\
\text { with high trait value }\end{array}$ \\
Global histology score & 13 & $37-85$ & $4.3^{\mathrm{A}}$ & FVB \\
Glomerulosclerosis & 4 & $5-35$ & $2.8(3.8)^{\mathrm{A}, \mathrm{B}}$ & $\mathrm{B} 6$ \\
& 13 & $42-82$ & $4.5^{\mathrm{A}}$ & $\mathrm{FVB}$ \\
Tubular dilation/casts & 4 & $5-35$ & $2.9(3.7)^{\mathrm{A}, \mathrm{B}}$ & $\mathrm{B} 6$ \\
& 13 & $37-85$ & $4.3^{\mathrm{A}}$ & $\mathrm{FVB}$ \\
Tubular degeneration & 4 & $5-35$ & $2.3(3.0)^{\mathrm{B}}$ & $\mathrm{B} 6$ \\
& 13 & $37-80$ & $4.3^{\mathrm{A}}$ & $\mathrm{FVB}$ \\
Podocyte hypertrophy & 4 & $0-44$ & 2.4 & $\mathrm{~B} 6$ \\
& 13 & $32-89$ & $3.5^{\mathrm{A}}$ & $\mathrm{FVB}$ \\
BUN & 7 & $15-82$ & 2.8 & $\mathrm{FVB}$ \\
& 13 & $34-62$ & 2.0 & $\mathrm{FVB}$ \\
Proteinuria (age 3 wk) & 3 & $5-54$ & 1.9 & $\mathrm{~B} 6$ \\
& 2 & $74-112$ & 2.3 & $\mathrm{FVB}$ \\
Proteinuria (age 12 wk) & 13 & $54-64$ & 1.8 & FVB \\
\hline
\end{tabular}

AStatistically significant based on 10,000 permutations of phenotype on genotype. ${ }^{B}$ The lod scores in parentheses reflect lod scores on chromosome 4 after adjustment for linkage to chromosome 13. pitulated in murine models of HIVAN, where the development of nephropathy is profoundly influenced by genetic background (38, 44-46). By analysis of genetic linkage in crosses of mouse strains with contrasting susceptibility, we previously localized a major locus for HIVAN, called HIVAN1, to chromosome 3A1-A3 (45). Moreover, we found large variation in disease penetrance among inbred strains, implying the existence of additional HIVAN quantitative trait loci (QTLs) that could delineate shared mechanisms underlying susceptibility to HIVAN. Here we show that podocyte gene expression is heritable and that HIVAN susceptibility genes belong to a regulated transcriptional network that modulates expression of podocyte cytoskeletal proteins such as Myb9 as well as components of the glomerular filtration barrier. ponents would provide insight into pathogenic pathways leading to kidney failure in the setting of podocyte mutations.

Combining gene expression profiling with linkage analysis (expression quantitative trait locus [eQTL] mapping) has emerged as a powerful tool for elucidating molecular pathways downstream of disease-causing mutations (24-28). eQTL mapping studies have demonstrated that transcript abundance is an inherited trait that is influenced by local genetic variation in the proximity of the gene locus itself (cis-eQTLs) or by distant loci (trans-eQTLs) that modulate gene expression through chromatin remodeling, transcriptional regulation, or more often, by complex secondary mechanisms (24, 25). Identification of loci that influence both clinical phenotypes and transcript abundance can uncover molecular pathways that link sequence variation to disease (26). For example, given linkage of a gene expression trait and a clinical phenotype to the same locus, statistical models can determine whether the gene expression trait is driving the clinical phenotype or whether variation in the clinical phenotype produces changes in the gene expression trait, resulting in a secondary linkage signal. Thus, the statistical relationship among variation in DNA, gene expression, and clinical phenotypes is utilized to predict directionality among them and infer causal relationships driving the pathogenesis of disease. Because genes that share a common transregulator are likely to participate in a functionally related pathway, they can be used to build molecular networks underlying complex traits $(26,29,30)$. This approach has been successfully applied to identify genes underlying obesity and metabolic syndrome $(29,30)$.

Here we apply the eQTL mapping strategy to study mechanisms underlying genetic susceptibility to HIVAN. HIVAN, a major cause of kidney failure in HIV-1 infection, is characterized by collapsing glomerulopathy and microcystic tubular dilatation (31-35). HIV-1 disrupts multiple cellular pathways in podocytes, resulting in enhanced reentry into the cell cycle and loss of expression of signature proteins such as podocin or Synpo, ultimately resulting in proliferation or apoptosis (36-41). A role for host susceptibility factors is demonstrated by increased prevalence among patients of African ancestry, which is strongly attributable to variation in the MYH9 gene $(10,42,43)$ Host predisposition is also reca-

\section{Results}

Identification of new HIVAN susceptibility loci in a transgenic FVB/NJ $\times$ C57BL/6J F2 intercross. We previously generated a backcross (BC) between HIV-1 transgenic FVB/NJ (TgFVB) and CAST/EiJ (CAST) mice and identified a susceptibility locus for HIVAN on chromosome 3A1-3A3 (called HIVAN1) with CAST alleles associated with an increased risk of disease (45). To identify additional nephropathy susceptibility loci, we produced an F2 intercross between TgFVB and the C57BL/6J (B6) strain $(n=191)$ and performed a genome-wide analysis of linkage using 103 informative SNPs to detect loci influencing multiple nephropathy-related phenotypes (histologic injury score, proteinuria, and blood urea nitrogen [BUN]; see Table 1). In this independent cross, there was no evidence of linkage of nephropathy phenotypes to the HIVAN1 or the Myb9 loci (on chromosomes 3 and 15, respectively). However, we found significant linkage of histologic injury traits to chromosome 13A3-C2 (the HIVAN2 locus; peak lod score 4.3 at rs3023383; see Table 1). Linkage to this segment was significant for multiple components of histologic scores and suggestive for BUN and proteinuria. We also identified linkage of histology injury to the centromeric region of chromosome 4A1-A5 (HIVAN3 locus), where linkage was initially suggestive but achieved genome-wide significance after controlling for the effect of HIVAN2 (Table 1). The HIVAN2 and HIVAN3 loci accounted for $10 \%$ and $7 \%$, respectively, of the variance in glomerulosclerosis scores in this cross. Interestingly, at HIVAN2, the $\mathrm{FVB} / \mathrm{NJ}$ (FVB) allele conferred increased risk of renal injury with an additive effect, while at HIVAN3 the B6 allele conferred increased risk with a recessive effect. We also identified 2 suggestive intervals for proteinuria (chromosomes 2 and 12; Table 1). Together with the initial cross we previously reported (45), these data identify 3 distinct HIVAN susceptibility loci on chromosomes 3A1-A3 (HIVAN1), 13A3-C2 (HIVAN2), and 4A1-A5 (HIVAN3).

HIVAN1 and HIVAN2 influence Nphs2 transcript abundance. To determine whether these HIVAN susceptibility loci were associated with variation in podocyte gene expression, we examined renal expression of Nphs1, Nphs2, Cd2ap, Actn4, Pdxl, and kin of IRRE like (Kirrel) in TgFVB and protected F1 hybrids (F1; FVB $\times$ B6, FVB $\times$ 

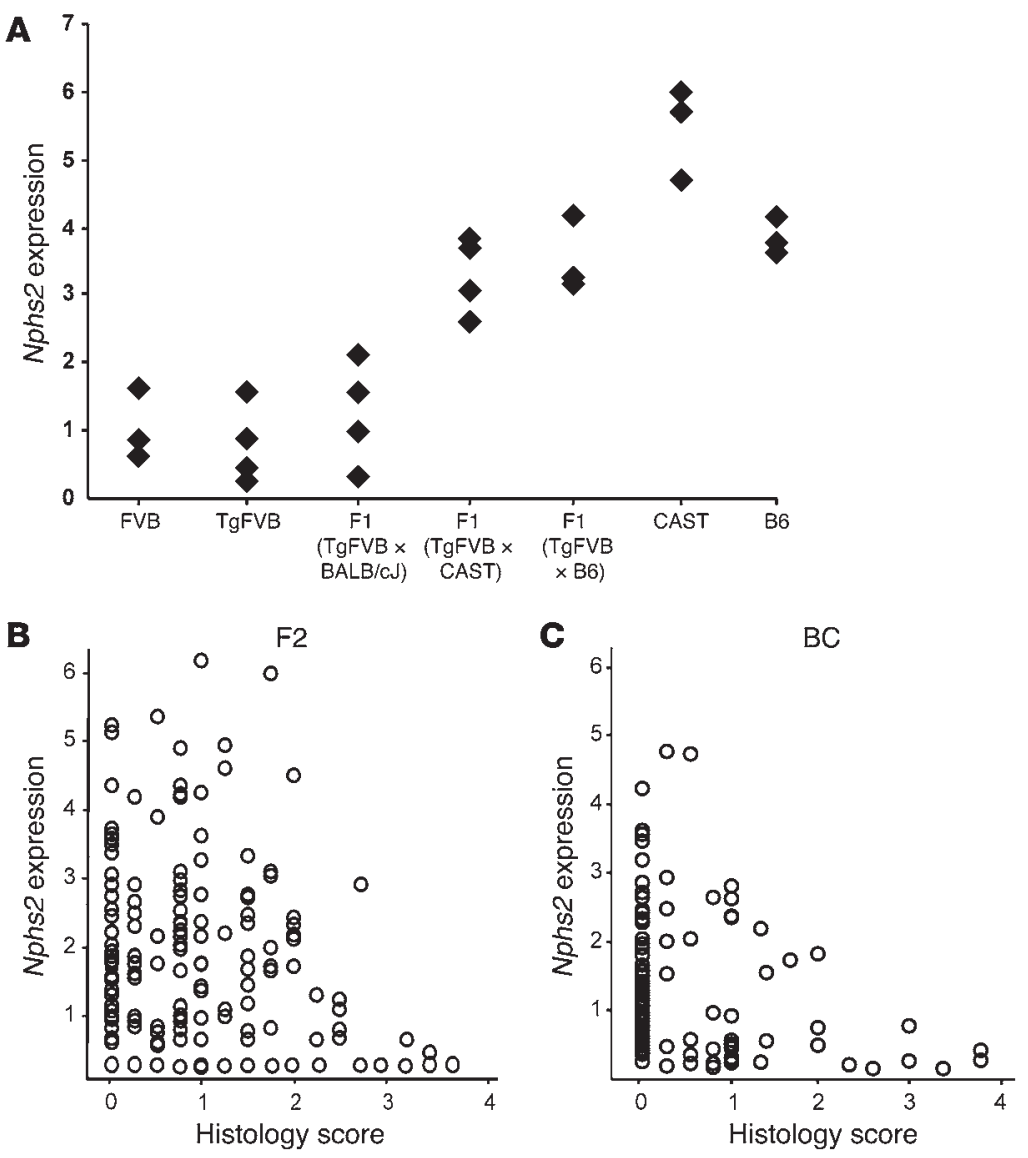

\section{Figure 1}

Variation in Nphs2 transcript abundance and its correlation with renal injury. (A) Interstrain variation in Nphs2 transcript abundance. (B and C) Distribution of podocin gene expression in HIVAN intercross (F2) and BC in relation to histologic injury score. Each point indicates data from an individual mouse. Expression levels in A-C are the ratio of expression level of each sample to that of an internal reference sample (FVB).

mosomes 1 and 13 as covariates in the analysis, we identified suggestive evidence for yet another $N p h s 2$ trans-eQTL on chromosome 3A1-A3 (lod score of 2.4 and 2.1 in $\mathrm{F} 2$ and $\mathrm{BC}$, respectively), an interval that corresponds to the HIVAN1 locus. The cis-eQTL and the trans-eQTLs on chromosomes 13 and 3 account for $40 \%, 10 \%$, and $4 \%$ of the variance in the Nphs 2 transcript level in the F2 cross and $45 \%, 12 \%$, and $5 \%$ of the variance in the $\mathrm{BC}$, respectively. The cis-eQTL and the HIVAN2 locus achieved genomewide significance based on published criteria as well as permutation analyses, whereas linkage to the HIVAN1 locus was suggestive in the 2 independent crosses. Moreover, there was complete overlap in the peak lod scores and the 95th percentile confidence interval for these eQTLs (Figure 2 and Table 1). Therefore, there was remarkable agreement in 2 independent mapping cohorts, demonstrating heritability of Nphs 2 transcript abundance and identifying 2 Nphs 2 trans-eQTLs that coincide precisely with the HIVAN1 and HIVAN2 loci.

To determine whether the overlap between eQTLs

CAST, and FVB $\times$ BALB/cJ; refs. 45, 46). Among these genes, only Nphs 2 expression demonstrated significant variation (ANOVA $P<0.0001$ ), with levels 3- to 4-fold higher among B6 or CAST F1 hybrids (Figure 1). Measurement in wild-type parental strains demonstrated that this variation was background dependent and unrelated to the presence of the HIV-1 transgene (Figure 1A). We next measured whole-kidney expression of $N p h s 2$ by quantitative PCR in $145(\mathrm{TgFVB} \times \mathrm{B} 6) \mathrm{F} 2$ intercross mice $(\mathrm{F} 2)$ and 123 previously produced $(\mathrm{TgFVB} \times \mathrm{CAST}) \mathrm{F} 1 \times \mathrm{FVB}$ BC mice (reported in ref. 45$)$. The level of $N p h s 2$ gene expression was continuously distributed in both cohorts and inversely related to renal injury, suggesting multifactorial determination (quadruplicate measurements; Figure 1, $\mathrm{B}$ and $\mathrm{C}$ ). The variance among mice with no evidence of histologic injury in these crosses was very high compared with the variance in genetically homogeneous strains, or F1s, implying strong genetic regulation of gene expression.

We next performed a genome-wide linkage analysis of $N p h s 2$ transcript abundance in the $\mathrm{F} 2$ and $\mathrm{BC}$ mice, with glomerulosclerosis treated as a covariate to adjust for secondary changes in transcript levels due to kidney injury. Remarkably, both cohorts identified the same loci contributing to variation in Nphs 2 gene expression. The first linkage signal localized to within $3 \mathrm{cM}$ of the $N p h s 2$ gene locus on chromosome $1 \mathrm{G} 3$, signifying the presence of a cis-eQTL (lod scores of 20.5 and 7.2 in F2 and BC mice, respectively; Figure 2, A and D). The second peak coincided with the HIVAN2 locus on chromosome 13A2-C2, identifying a trans-eQTL (lod score of 4.5 and 3.3 in $\mathrm{F} 2$ and $\mathrm{BC}$ mice, respectively; Figure 2, B and E). In addition, after taking glomerulosclerosis and the eQTLs on chro- and nephropathy loci was due to chance, we performed a standard test of linkage versus pleiotropy, in which we tested 2 alternative models: the hypothesis that 2 distinct genes that are closely located within the QTL locus influence each trait separately (linked QTL model) and the null hypothesis that 1 gene in the interval influences both traits (pleiotropic model) (47). Based on this test, the best locations for the QTL position in the 2 models were statistically indistinguishable $(P=0.6$ and $P=0.3$ for the HIVAN1 and HIVAN2 loci, respectively). As a second statistical approach, simulation studies under the assumption of independence between nephropathy and Nphs 2 traits indicated that the probability of partial or complete overlap of confidence intervals in 2 crosses by chance was $1 / 200$ and $1 / 5,000$, respectively. Therefore, we found no evidence that 2 distinct genes in the HIVAN loci affect HIVAN and Nphs 2 expression separately.

To exclude any confounding effects of kidney injury on findings, we re-examined the Nphs 2 expression data in the subset of mice with normal histology and no proteinuria. The evidence for Nphs 2 cis- and trans-eQTLs remained highly significant, with consistent phenotypic effects across both mapping cohorts $(n=62$ in BC and $n=59$ in F2 cross; Figure 3, A-C). This demonstrated that these intervals influence Nphs 2 expression independently of glomerulosclerosis. Moreover, we found that the nephropathy-predisposing alleles were associated with either high (HIVAN2) or low (HIVAN1) Nphs 2 expression. These data, combined with the absence of linkage of glomerulosclerosis to the strong Nphs 2 cis-eQTL (lod score less than 0.5 in each cross; Figure $2 \mathrm{~A}$ ) indicate that variation in Nphs 2 expression is not a causal factor in the development of glomerulo- 
A
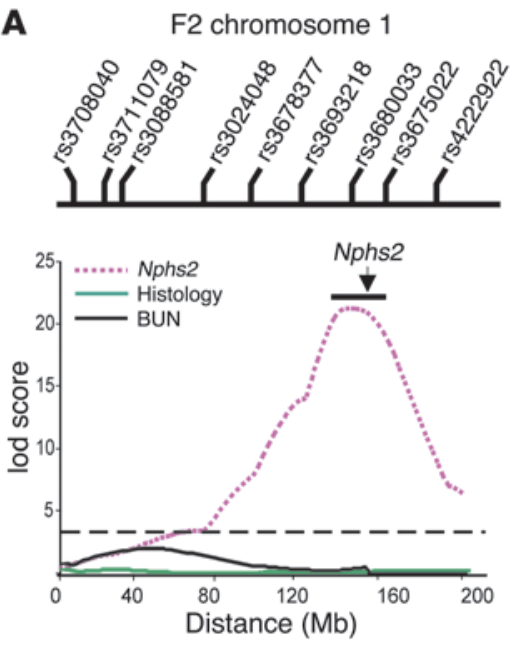

D

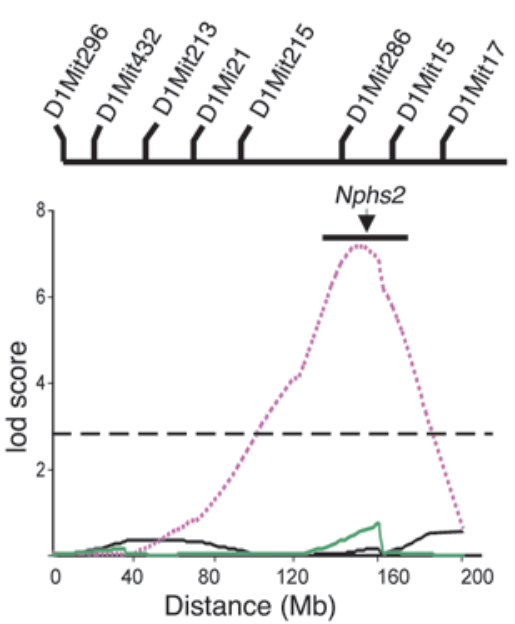

B
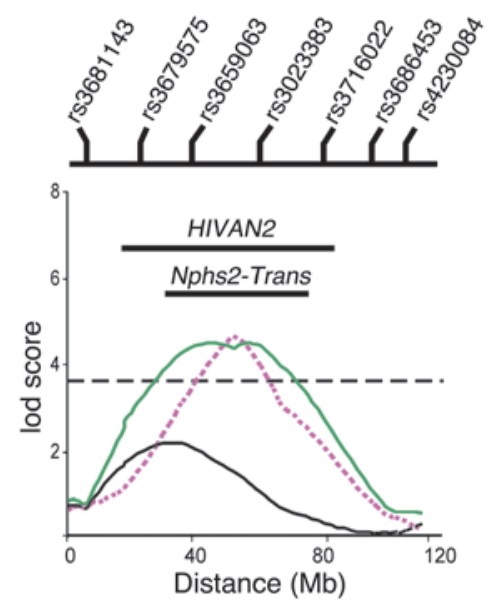

E
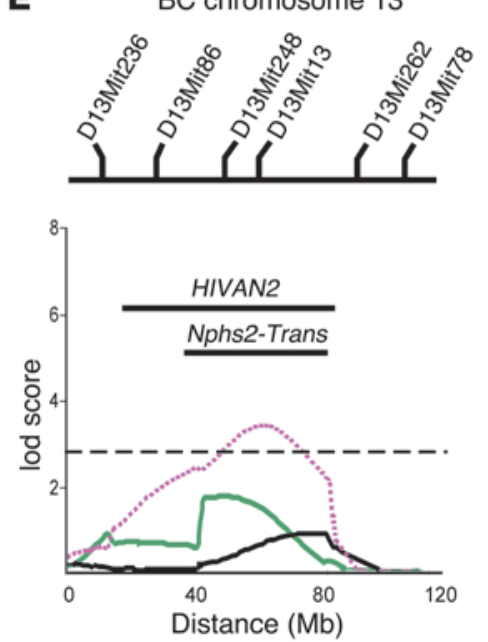

C

F2 chromosome 3
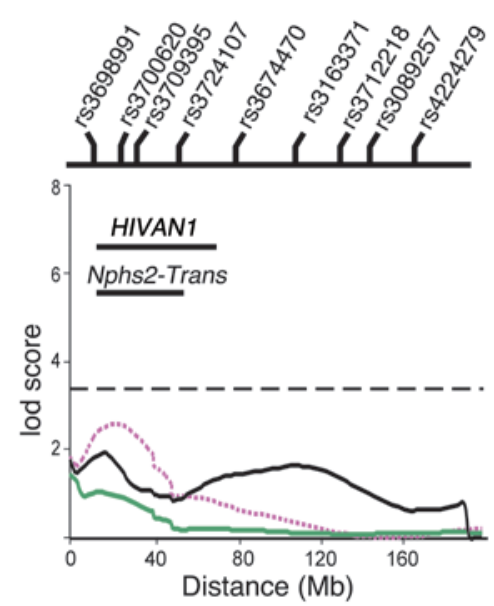

$\mathbf{F}$

BC chromosome 3
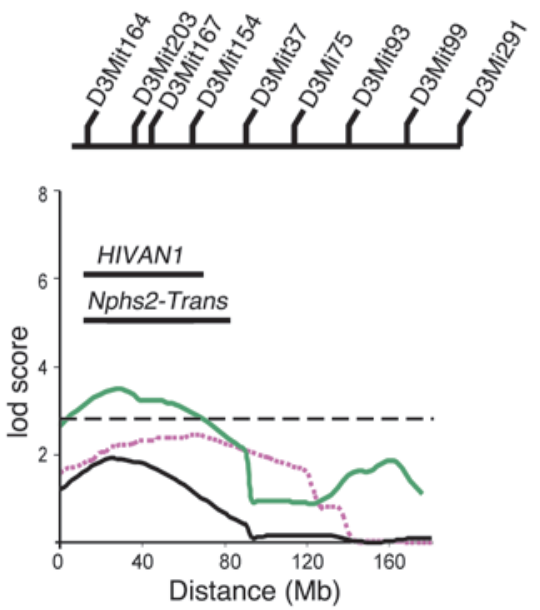

Figure 2

Lod plots for Nphs2 eQTLs in the F2 and BC cohorts. (A and D) Linkage of Nphs2 expression to the Nphs2 locus on chromosome 1 in F2 and BC. (B and E) Linkage of Nphs2 expression to the HIVAN2 locus on chromosome 13 in F2 and BC. (C and F) Suggestive linkage of Nphs2 expression to the HIVAN1 locus on chromosome 3 in F2 and BC (after adjustment for the chromosomes 1 and 13 eQTLs). The positions of informative markers are shown above each plot. The dashed horizontal lines indicate the thresholds for genome-wide significance (3.6 in F2 and 2.9 in BC). The bars above the lod plot indicate the 95th percentile confidence interval for each trait locus. The location of Nphs 2 is shown above the lod plot for chromosome 1. The 95th percentile confidence intervals for the Nphs2 eQTLs were: on chromosome 1, 142-162 (F2) and 140-174 $\mathrm{Mb}(\mathrm{BC})$; on chromosome 13, 36-79 Mb (F2) and 32-84 Mb (BC); on chromosome 3, 6-48 Mb (F2) and 6-64 Mb (BC).

sclerosis. The alternative explanation is that the linkages of $N p h s 2$ expression to HIVAN loci represent reactive or regulatory responses to perturbations produced by HIVAN susceptibility alleles. The presence of such a regulatory response can be prospectively tested by determining whether introduction of a known genetic perturbation (i.e., the Nphs2 cis-eQTL) results in altered expression of additional podocyte genes and whether this response is similar to genetic profile elicited by HIVAN susceptibility alleles.

Tightly linked sequence variants at the Nphs 2 locus are associated with a 3-fold difference in transcript level, producing perturbations in other podocyte genes. We initially investigated the Nphs 2 cis-eQTL because it contributes a 3- to 4-fold difference in transcript level and can validate the hypothesis of a reactive response in podocyte genes. To verify that Nphs 2 expression is affected by one or more cis- variants, we sequenced the $N p h s 2$ gene $(2.5-\mathrm{kb}$ sequence upstream of the start codon, the exons, and $3^{\prime}$ UTR) in 19 inbred strains, including the 3 parental strains with contrasting expression profile. We identified a larger than expected number of sequence variants among the strains tested, including a total of 103 SNPs and 13 insertions/deletions. Among these were 4 non-synonymous SNPs and 2 exonic insertions/deletions (s 2-4). The SNP density in the Nphs 2 locus was 1 variant per approximately 36 bp surveyed, which is 8 -fold higher than the genome-wide average for inbred strains (48). Phylogenetic analysis demonstrated that these SNPs were not randomly distributed but clustered into 3 major haplotypes that corresponded to the ancestral genomes of the laboratory mouse (Mus musculus domesticus, Mus musculus musculus, and Mus musculus castaneus; Figure 4A). The M.m. musculus haplotype differed from the M.m. domesticus and M.m. castaneus strains by 60 and 77 sequence variants, respectively, while the latter 2 were dis- 
A

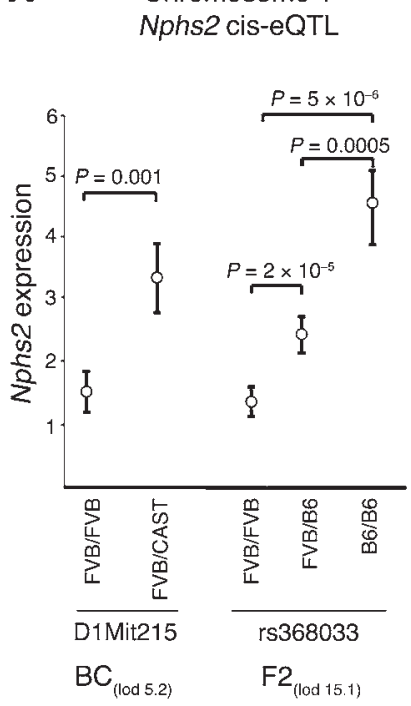

B Chromosome 13
Nphs2 trans-eQTL (HIVAN2)

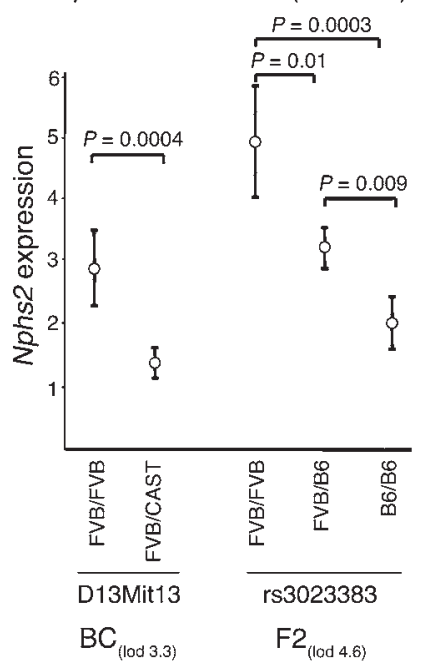

C Chromosome 3 Nphs2 trans-eQTL (HIVAN1)

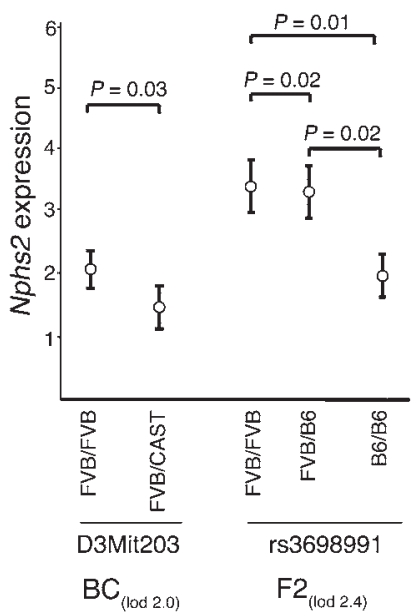

Figure 3

Nphs2 expression levels at each eQTL in BC $(n=62)$ and F2 $(n=59)$ mice with no evidence of renal disease. (A) Cis-eQTL on chromosome 1. (B and C) Trans-eQTL on chromosomes 13 (B) and 3 (C). The expression value for the HIVAN1 locus on chromosome 3 was adjusted for chromosome 1 and chromosome 13 eQTLs. Expression levels in A-C are the ratio of the expression level of each sample to that of an internal reference sample (FVB). The genotypes and markers at the locus are indicated on the $x$ axis. Data are mean \pm SEM.

tinguished by 31 sequence variants. In addition, we observed a substantial number of private intrasubspecific variants. Notably, we found a 30-bp in-frame deletion in exon 1 in the B6, SJL/J, and CFW strains that was absent in other inbred strains, including the wild-derived strains representative of the ancestral mouse genomes ( $\mathrm{PWD} / \mathrm{PhJ}$, CAST, and WSB/EiJ), indicating that the deletion occurred during the derivation of inbred strains. The combination of high SNP density and the occurrence of new intrasubspecific SNPs indicate that the Nphs2 sequence has experienced an unusually high mutation rate across the mouse lineage. We calculated the ratio of non-synonymous (Ka) to synonymous (Ks) nucleotide substitution rates to determine whether the high polymorphism rate can be explained by positive selection of Nphs2. However, the $\mathrm{Ka} / \mathrm{Ks}$ ratio across all amino acid sites in $N p h s 2$ was $0.33(\mathrm{Ka} / \mathrm{Ks}>1$ is indicative of positive selection) and a whole gene likelihood ratio test showed no evidence to support positive selection $(P=0.30)$.

We next performed an association study of the 3 Nphs2 haplotypes with transcript abundance (Figure 4B). The Nphs2 haplotypes explained $40 \%$ of the variance among inbred strains. The strains with the M.m. musculus haplotype (FVB/NJ [FVB], C3H/HeJ, $\mathrm{CBA} / \mathrm{J}, \mathrm{A} / \mathrm{J}, \mathrm{DBA} / 2 \mathrm{~J}, \mathrm{RIIIS} / \mathrm{J}$, and BALB/cJ) demonstrated 3-fold lower expression of Nphs 2 compared with the strains with the M.m. domesticus haplotype (B6, C57BL/10J, SJL/J, CFW, and 129/SvEv; $P=3 \times 10^{-12}$ ), while there were no significant differences between strains carrying the M.m. domesticus and M.m. castaneus haplotypes $(P=0.1)$. Western blot analysis in the representative strains confirmed gene expression data classifying the same strains into highand low-expression groups (Figure $4 \mathrm{C}$ ). We also conducted $5^{\prime}$ and $3^{\prime}$ RACE in B6 and FVB strains but found no evidence of alternative transcripts. These data provide clear evidence that the large variance in Nphs2 expression among inbred strains is attributable to one or more sequence variants within this locus.
To study the trans-regulatory effects of the Nphs2 cis-eQTL and test the hypothesis of a networked response in podocyte genes, we examined B6-chr01 ${ }^{\mathrm{A}}$-consomic strains, in which chromosome 1 from the $\mathrm{A} / \mathrm{J}$ strain (low-expression haplotype) was substituted into the B6 (high-expression haplotype) genome (Figure 5A). As predicted by the linkage and inbred strain data, substitution of chromosome 1 from $\mathrm{A} / \mathrm{J}$ into the $\mathrm{B} 6$ genome resulted in a primary reduction in $N p h s 2$ expression (3-fold difference for the $\mathrm{B} 6-\mathrm{chr} 01^{\mathrm{A}}$ strain compared with the $\mathrm{B} 6$ reference strain; $P=10^{-7}$ ) but no overt evidence of nephropathy. We next examined whether this primary decrease in Nphs2 is associated with altered expression of its known interacting partners (Nphs1, Cd2ap, and Kirrel) as well as other podocyteexpressed genes (Plce1, Myb9, Wilms tumor 1 homolog [Wt1], and Synpo). Compared with the B6 reference strain, B6-chr01 $1^{\mathrm{A}}$ exhibited 1.3- to 2.8-fold increased expression of Myb9 $\left(P=5 \times 10^{-7}\right)$, Synpo $(P=0.006)$, Nphs1 $(P=0.04), P l c e 1(P=0.004)$, and $\operatorname{Kirrel}(P=0.005)$ but no changes in Cd2ap or Wt1 transcript (Figure $5 \mathrm{~A}$ ). Importantly, although we tested only 3 intervals and 1 consomic substitution, the $M y b 9$ and Plce1 comparisons reached genome-wide significance levels for mapping in 20 consomic strains $(P<0.004)(49)$. Thus, in response to a primary genetic perturbation in $N p h s 2$, we observed a significant augmentation in expression of multiple podocyte genes involved in the composition and regulation of the slit diaphragm. Because the altered transcripts are not encoded on chromosome 1, variation in their expression can only be attributed to trans-regulation. Moreover, since the B6-chr01 ${ }^{\mathrm{A}}$ mice did not have nephropathy, these findings are independent of the confounding effects of glomerulosclerosis and establish normative data about podocyte response to a primary decrease in $N p h s 2$ expression.

HIVAN1 and HIVAN2 susceptibility alleles activate the same podocyte regulatory pathway as the hypomorphic Nphs 2 allele. We next determined whether HIVAN susceptibility genes also activate the same regulated pathway by studying 2 inbred strains harboring genomic 
A

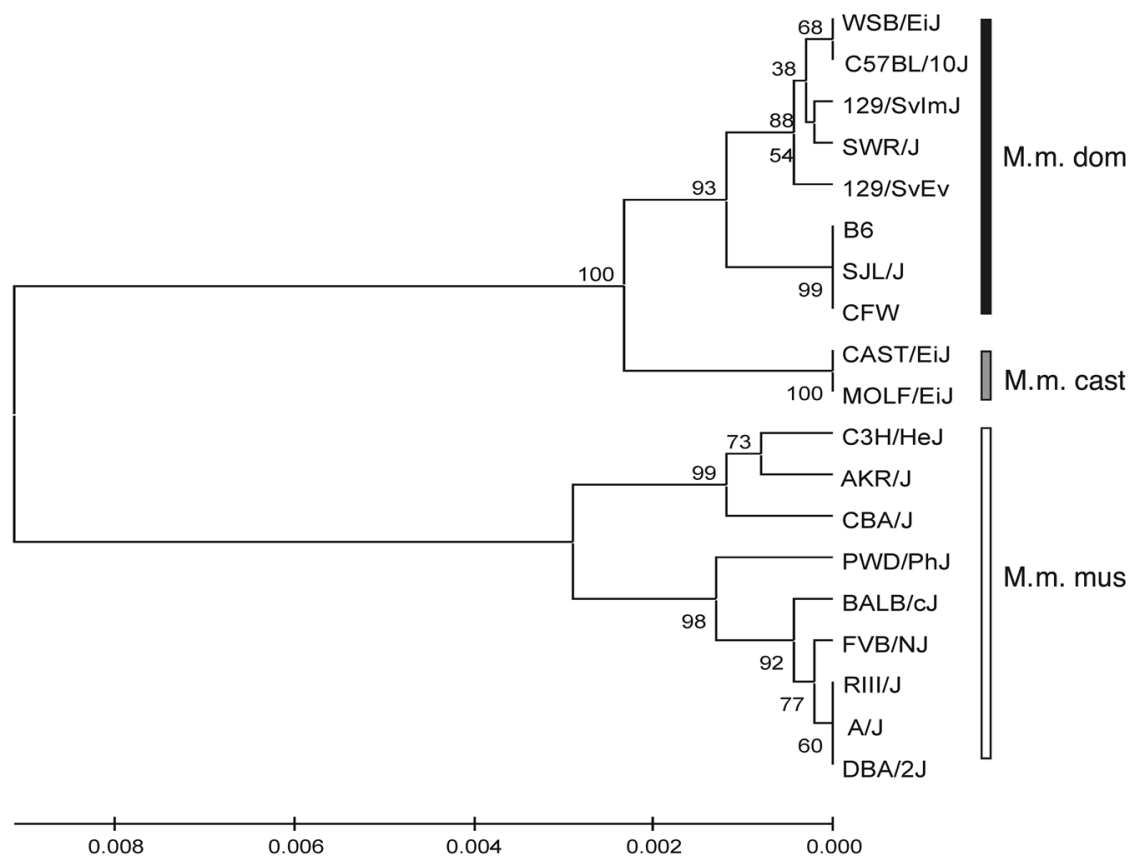

B

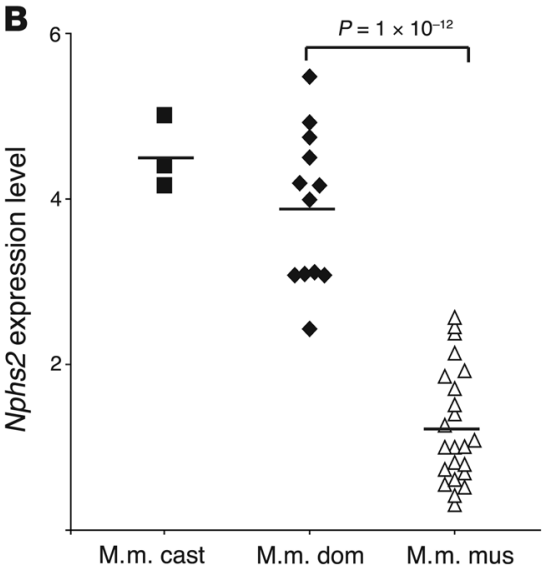

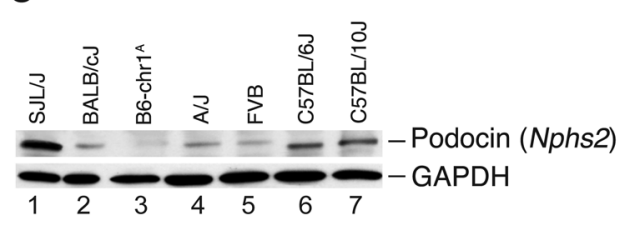

\section{Figure 4}

Podocin (Nphs2) level is associated with mouse ancestral haplotype. (A) Phylogeny of the Nphs2 sequence identified 3 major clades among 19 inbred strains. Numbers indicate the percentages of replicate trees in which the associated taxa clustered together in the bootstrap test (10,000 replicates). Scale indicates branch lengths and the evolutionary distances, measured as the number of base substitutions per site. (B) Nphs2 expression level in inbred mice was significantly associated with haplotype. Individual points indicate Nphs2 levels in mice of various strains, grouped by ancestral haplotype, with means shown for each group. Expression levels are shown as the ratio of the expression level of each sample relative to that of an internal reference sample (FVB). (C) Western blot analysis of podocin levels in the kidney of representative mouse strains confirmed quantitative PCR data. Positions of podocin and GAPDH (loading control) are indicated. M.m. mus, M.m. musculus; M.m. cast, M.m. castaneus; M.m. dom, M.m. domesticus. substitution encompassing the Nphs2 trans-eQTLs (HIVAN1 and HIVAN2 loci). To examine the trans-regulatory effects exerted by the HIVAN2 locus, we studied B6-chr13 ${ }^{\mathrm{A}}$ mice, in which chromosome 13 from the A/J genome was substituted into the $\mathrm{B} 6$ genome. These consomic strains were suitable because A/J and FVB strains both have the low-expression haplotypes at the cis-eQTL, and Nphs2 expression levels are nearly identical between them (Figure 4), suggesting that they also share the same alleles at the Nphs 2 transeQTL. As predicted, B6-chr $13^{\mathrm{A}}$ mice demonstrated a 2-fold increase in Nphs 2 transcript abundance $\left(P=2 \times 10^{-8}\right.$ versus the $\mathrm{B} 6$ strain; Figure $5 \mathrm{~B})$. In addition, this genomic substitution was characterized by 1.3- to 2.7-fold increased expression of $M y h 9(P=0.008)$, Nphs1 $\left(P=5 \times 10^{-6}\right), C d 2 a p(P=0.0008)$, Kirrel $(P=0.017)$, Plce1 $(P=0.008)$, and Synpo $(P=0.03)$ but no changes in $W t 1$ expression. The Myb9 and Nphs 1 comparisons reached genome-wide significance levels for mapping in 20 consomic strains (49). These data confirmed our initial hypothesis that HIVAN susceptibility alleles modify podocyte gene expression independent of HIV-1 and the Nphs2 cis-eQTL. Moreover, this augmented expression closely resembles the profile observed in $\mathrm{B} 6-\mathrm{chr} 01^{\mathrm{A}}$ mice, which have a primary decrease in Nphs 2 expression. This similarity in gene expression response suggests that the HIVAN2 susceptibility alleles produce a genetic lesion that resembles a reduction of $N p h s 2$ expression and that the Hivan2, Myb9, and Nphs2 genes belong to a common regulatory pathway.

We next examined the expression profile elicited by HIVAN1 susceptibility alleles. We generated HIVAN1 congenic mice by introgressing approximately $52 \mathrm{Mb}$ of the CAST genome at the HIVAN1 locus into the FVB genetic background (FVB-HIVAN1 ${ }^{\text {CAST }}$ mice). HIV-1 transgenic FVB-HIVAN1 ${ }^{\text {CAST }}$ mice develop accelerated renal failure compared with TgFVB, but their nontransgenic counterparts are viable and phenotypically normal (50). However, examination of renal gene expression in nontransgenic congenic mice demonstrated that CAST alleles at the HIVAN1 locus result in a significant reduction in baseline expression of $N p h s 2(P=0.04)$ and Kirrel $(P=0.001)$ compared with the FVB strain (Figure 5C). Moreover, CAST alleles were also associated with significantly increased expression of Myh9 $(P=0.01)$ but no changes in Cd2ap, 
A

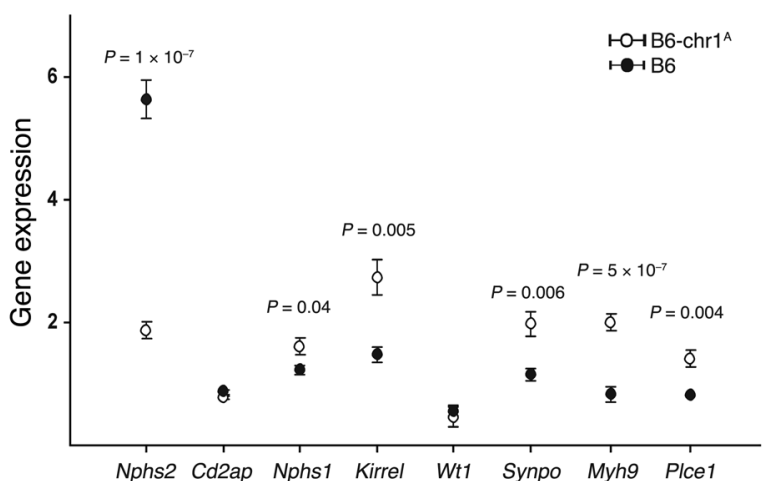

C

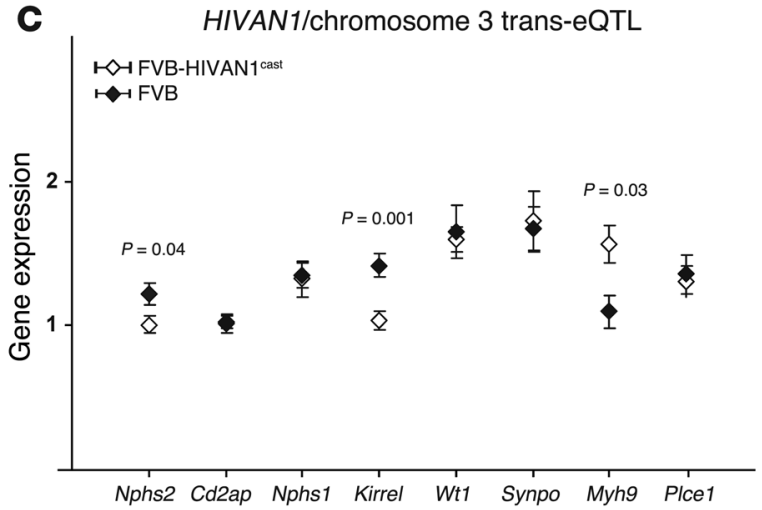

B

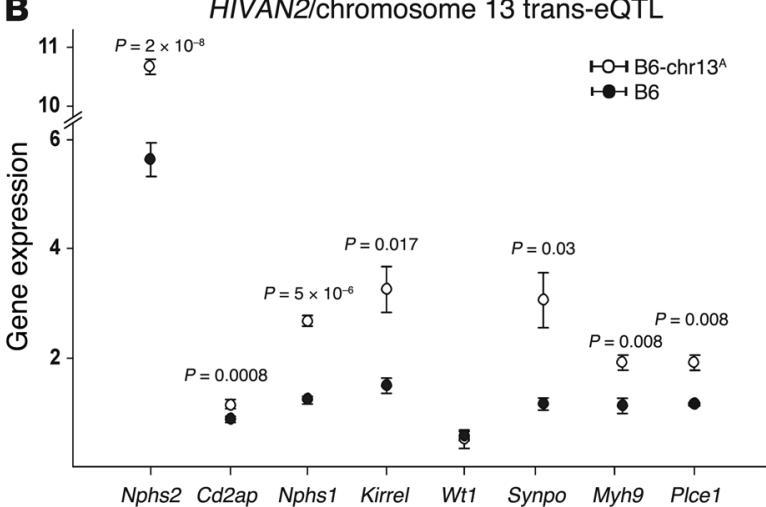

D

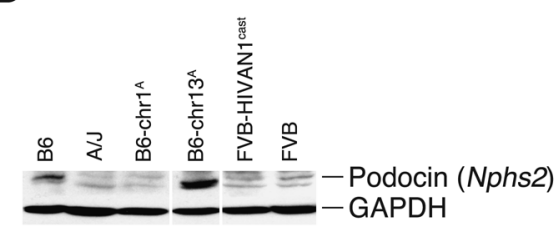

\section{Figure 5}

Distinct expression profile of podocyte genes in the congenic and consomic strains harboring Nphs2 eQTLs compared with their respective reference strains. (A) Nphs2 cis-eQTL. (B) HIVAN2/chromosome 13 trans-eQTL. (C) HIVAN1/chromosome 3 trans-eQTL. Data are mean \pm SEM. Expression levels in $\mathbf{A}-\mathbf{C}$ are shown as the ratio of the expression level of each sample to that of an internal reference sample (FVB). (D) Western blot analysis demonstrated concordance of quantitative PCR and protein expression levels for podocin. $P$ values for comparison with reference strains are shown for each transcript. B6 $(n=10), \mathrm{FVB}(n=20), \mathrm{B} 6-\operatorname{chr}^{\mathrm{A}}(n=6), \mathrm{B} 6-\operatorname{chr}_{13}^{\mathrm{A}}(n=4)$, and FVB-HIVAN1 ${ }^{\mathrm{CAST}}(n=20)$.

Nphs1, Plce1, Synpo, or Wt1 transcript levels. This gene expression profile is qualitatively different from the response observed at the Nphs 2 cis-eQTL or the HIVAN2 locus, suggesting that the HIVAN1 susceptibility allele produces a different defect in podocytes.

Altogether, after interrogating 3 genomic segments (corresponding to $372 \mathrm{Mb}$ or $\sim 12.3 \%$ of the mouse genome), we identified 16/24 transcripts with significant differences in expression at $P<0.04$, whereas 1 significant difference would have been expected by chance (false discovery rate $=0.06$ ). Moreover, each interval tested was significant for at least 3 expression traits and, conversely, $6 / 8$ interrogated transcripts were significant in at least 2 intervals. Finally, 7/24 comparisons (including 2 for $N p h s 2)$ reached genome-wide significance for mapping in consomic strains $(P<0.004$; ref. 49$)$. The possibility of multiple independent eQTLs that influence each podocyte transcript separately cannot be formally excluded without fine mapping of each congenic/consomic interval. However, based on the genome-wide density and frequency of trans-eQTLs reported in one of the largest studies in the mouse (28), we estimate that the probability of chance detection of 5 genome-wide significant trans-eQTLs that are independent of Nphs 2 trans-eQTLs is $3 \times 10^{-5}$. Thus, the most parsimonious explanation for our findings is that the same eQTL within each region jointly influences expression of Nphs 2 and other podocyte genes.
To complete these studies, we determined whether the podocyte expression profile is modified in the presence of HIV-1 by examining HIV-1 transgenic FVB-HIVAN1 ${ }^{\text {CAST }}$ and TgFVB mice. To minimize secondary effects of glomerulosclerosis on gene expression, ten 6-week-old transgenic mice of each genotype were matched for age, gender, and degree of renal injury (range of 9\%-55\% glomerulosclerosis). HIV-1 resulted in reduced expression of Myb9, Nphs1, and Wt1 in both genotype groups. On the other hand, we found a reciprocal change in the $C d 2 a p$, Synpo, Plce1, and Kirrel response according to HIVAN1 genotype (Figure 6). Compared with their nontransgenic counterparts, HIV-1 transgenic mice with the FVB alleles (protective) exhibited significantly increased expression of Kirrel $(P=0.01)$ and $C d 2 a p$ $(P=0.001)$ and no change in Synpo or Plce1. In contrast, mice harboring the nephropathy-prone CAST alleles demonstrated no change in Kirrel or Cd2ap transcript levels and a significant decrease in Synpo $(P=0.004)$ and Plce1 $(P=0.01)$ expression. These data remained significant after adjustment for degree of glomerulosclerosis and HIV-1 transgenic status, suggesting that interaction of HIV-1 with HIVAN1 alleles selectively blunts upregulation of Kirrel and Cd2ap while promoting downregulation of Synpo and Plce1. These data demonstrate that the podocyte gene expression is strongly modified by the presence of HIV-1 and by underlying HIVAN1 genotype. 

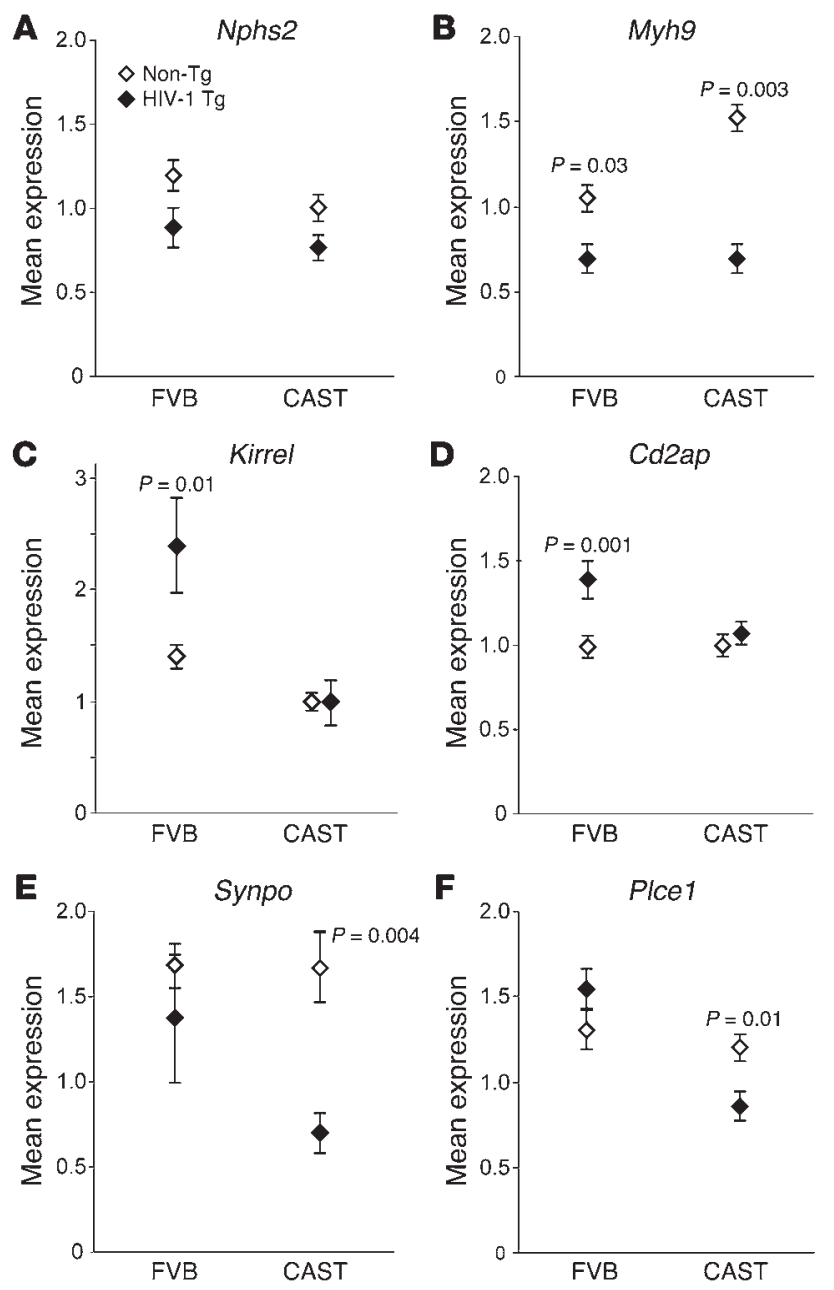

$\mathbf{F}$
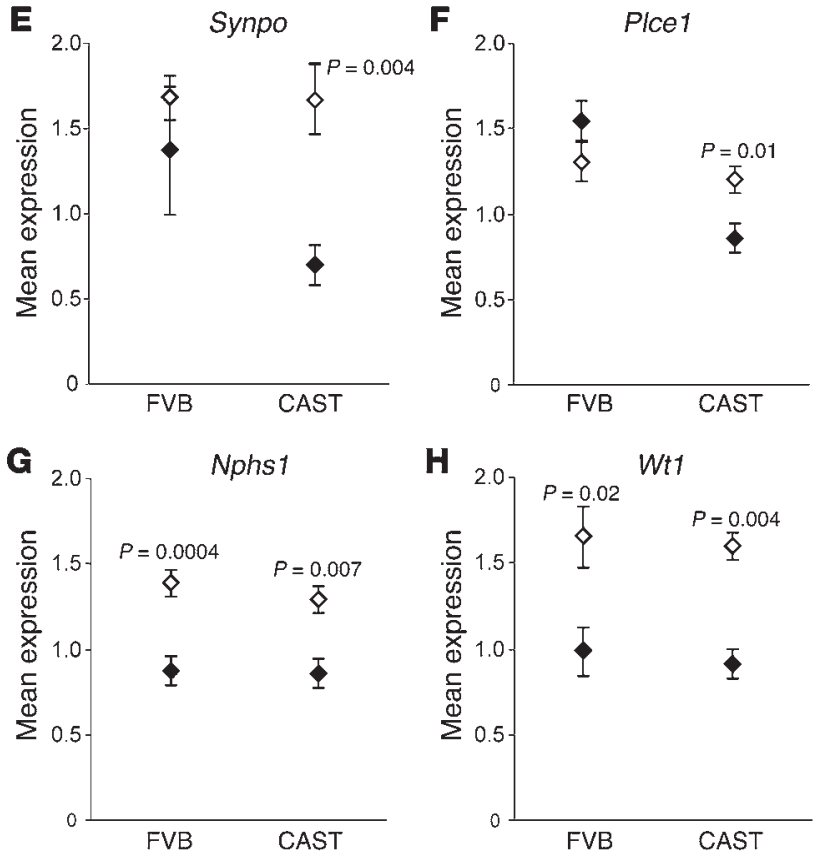

\section{Discussion}

In the present study, we demonstrate that genetic susceptibility to HIVAN is a latent state characterized by downstream perturbations in podocyte expression profile. We started with identification of 2 QTLs for HIVAN and demonstrated strong heritability of Nphs 2 transcript abundance across inbred strains with different susceptibility to HIVAN. Studies in 2 independent mapping cohorts demonstrated that variation in Nphs 2 expression is controlled a cis-eQTL and 2 trans-eQTLs. While the Nphs 2 cis-eQTL did not influence the development of glomerulosclerosis, the trans-eQTLs both coincided with susceptibility loci for HIVAN (HIVAN1 and HIVAN2), suggesting identity between genes influencing Nphs 2 transcript abundance and nephropathy. Because the

\section{Figure 6}

Expression of podocyte genes is altered by the HIVAN1 genotype and further modified in the presence of the HIV-1 transgene. The HIVAN1 genotype is shown on the $x$ axis (FVB, protective allele; CAST, susceptible allele). The expression levels (mean \pm SEM) of each podocyte transcript in wild-type (non-Tg, open symbols) and HIV-1 transgenic (filled symbols) are shown. Significant $P$ values for comparison between transgenic and nontransgenic mice within the same genotype group are indicated. While Nphs2 (A), Myh9 (B), Nphs1 (G), and Wt1 (H) showed downregulation in the presence of HIV regardless of genotype, there was a differential response to the HIV-1 transgene according to HIVAN1 genotype for Kirrel (C), Cd2ap (D), Synpo (E), and Plce1 (F) expression. Expression levels are shown as the ratio of the expression level of each sample to that of an internal reference sample (FVB). FVB $(n=20), \operatorname{TgFVB}(n=10)$, FVB-HIVAN1CAST $(n=20)$, and TgFVB-HIVAN1 ${ }^{\text {CAST }}(n=10)$.

pattern of the linkage data did not support a causal relationship between variation in Nphs2 expression and glomerulosclerosis, we inferred that the transregulation of Nphs 2 must represent reactive or compensatory changes secondary to genetic lesions introduced by the HIVAN susceptibility alleles. This suggested that expression of podocyte genes is networked and responsive to genetic perturbations. We validated this hypothesis by examining the transcriptional response to the naturally occurring $N p h s 2$ hypomorphic allele, demonstrating significant upregulation of multiple podocyte genes whose products have direct and indirect physical association with Nphs2 (Nphs1, Kirrel, Myb9, Plce1, and Synpo). Furthermore, nontransgenic HIVAN1 congenic and chromosome 13-consomic mice demonstrated upregulation of the same podocyte transcripts, placing murine HIVAN susceptibility genes within the same regulatory network that includes $N p h s 2, M y b 9$, and related components of the glomerular filtration. The identification of distinct but related molecular phenotypes for HIVAN1 and HIVAN2 further suggested that the underlying susceptibility genes introduce different lesions within this common regulatory pathway.

These data can be interpreted without the confounding effects of glomerulosclerosis or HIV-1 gene products, demonstrating that podocytes can modulate the expression of key components of the glomerular filtration barrier in response to genetic lesions. This concept can explain the seemingly contradictory finding that HIVAN susceptibility alleles were associated with both low (HIVAN1) and high (HIVAN2) Nphs2 expression. These differing expression profiles likely represent different modes of a regulatory pathway that is responding to distinct genetic perturbations introduced by HIVAN1 and HIVAN2 susceptibility alleles. Additional studies can determine whether this altered podocyte gene expression profile is merely reactive or indicative of a compensatory response that enables restoration of podocyte homeostasis. It is also important that upon introduction of HIV-1, HIVAN1 susceptibility alleles produced a very different gene expression profile compared with protective alleles, suggesting that gene-environment (e.g., viral genome) interactions disturb the regulatory network and produce the dysregulated podocyte phenotype that contributes to the development of nephropathy. One can postulate that similar events occur in the presence of MYH9 susceptibility alleles in humans, initiating the development of nephropathy. This hypothesis can be tested by examination of Myb9 haploinsufficient mice, which are phenotypically normal $(51,52)$, to determine whether they display a renal molecular signature similar to the 
congenic/consomic mice studied here and whether they develop nephropathy with appropriate environmental insults. Moreover, Ratelade et al. have recently identified modifier loci influencing proteinuria and kidney function in Nphs2-null mice and further demonstrated that maternal-fetal factors also profoundly modify these phenotypes (53). The nephropathy QTLs in the Nphs2-null mice are distinct from the HIVAN susceptibility loci but also include some podocyte-expressed genes (Synpo2 on chromosome 3 and Iggap1 on chromosome 7). Thus, it would be interesting to know whether the modifier loci identified in Nphs2-null mice control the expression of other podocyte genes.

Our eQTL study provides a framework for systematic reconstruction of the molecular network connecting to HIVAN susceptibility alleles, $M y$ h9, and other nephropathy-associated genes. For example, comprehensive renal expression profiling can uncover differentially expressed genes that the congenic/consomic mice have in common, identifying critical components of this regulatory pathway, while mapping studies can determine whether additional trans-eQTLs (in the substituted segments or elsewhere in the genome) influence the expression of podocyte transcripts, dissecting intermediate nodes in the network. In addition, genome-wide expression profiling in the F2 and BC mapping cohorts can identify the full network of genes that are transregulated by HIVAN loci, detecting causal mediators of disease and HIV-1-induced perturbations in these pathways. This approach can also identify cis-eQTLs that localize to the HIVAN1 and HIVAN2 intervals, thereby isolating strong positional candidates for nephropathy. Similar analytic approaches have recently been applied to dissect other complex traits, such as a chromosome 1 QTL associated with multiple metabolic- and obesity-related traits, where expression traits linking to this interval were decomposed into causal or reactive phenotypes, leading to identification of multiple interconnected genes that mediate disease $(29,30)$.

Although the HIVAN1 and HIVAN2 loci contain several transcription factors, none have a predicted binding site on the Nphs 2 promoter. Conversely, none of the transcription factors that are known to regulate Nphs2 map to HIVAN loci (Foxc2 is on chromosome 8 [ref. 54] and $L m x 1 b$ is on chromosome 2 [ref. 55]). These data are consistent with published eQTL studies that have indicated that trans-eQTL intervals encode many different gene classes, such as transcription factors, microRNAs, ligands, receptors, enzymes, substrates, or subunits of protein complexes that can affect their target transcript via a context-dependent mechanism $(26,56,57)$. These studies have also shown that functional variants underlying cis-eQTLs often involve multiple linked polymorphisms resulting in haplotype effects (58-60). This situation is illustrated by the analysis of the Nphs 2 cis-eQTL, in which the large number of sequence variants differentiating haplotypes with a contrasting expression profile suggested the potential for multiple functional alleles. It is also noteworthy that the $26-\mathrm{Mb}$ region encompassing the Nphs2 locus is enriched for genes related to immunity, defense, and olfaction, which are subject to strong positive selection and rapid evolution among most species. We found no evidence for positive selection of Nphs2 based on absence of excess non-synonymous substitutions. Additional sequence analysis of neighboring genes will be required to determine whether positive selection operated on other genes in this region, providing a permissive setting for accumulation of sequence variants in Nphs2 (61). These complexities of the mouse Nphs 2 locus present several opportunities for studying gene regulation. Because identified variants are associated with substan- tial in vivo differences in transcript and protein abundance, they offer an attractive catalog of functional candidates whose individual roles can be dissected by traditional promoter analysis. In particular, among the $116 \mathrm{Nphs} 2$ sequence variants we describe, 3 missense variants and a 21-bp deletion upstream of the transcription start site in the low-expression haplotype are promising functional candidates (s 2-4). Conversely, promoter segments devoid of SNPs may point to evolutionary conserved regulatory modules that direct podocyte-specific expression.

Our eQTL study presents a survey of the podocyte transcriptional response to nephropathy predisposing alleles, demonstrating the importance of underlying genotype and environment in interpreting the relationship of the gene expression profile to nephropathy. These studies outline a framework for reconstruction of the molecular networks connecting diverse glomerulopathy-associated genes. Identification of podocyte transregulators and their associated network will provide insight into the pathogenesis of multiple forms of primary and secondary nephropathy.

\section{Methods}

Animal breeding. All inbred strains (including consomic lines) were purchased from The Jackson Laboratory. The HIV-1 transgenic mouse line $\mathrm{TgN}$ (pNL43d14)26Lom 26 (TgFVB) was produced on the inbred FVB/N genetic background and has been extensively characterized $(44,45)$. The $\mathrm{TgFVB} \times(\mathrm{FVB} \times \mathrm{CAST}) \mathrm{F} 1 \mathrm{BC}$ has been previously reported (45). A second mapping cohort was produced for this study by intercrossing TgFVB $\times$ B6 F1 hybrids (F2 cohort) and phenotyped at 12 weeks of age. Animals that were moribund were euthanized prior to this end point. As before (45), 3 primary phenotypes related to renal disease were characterized: renal histology, BUN, and proteinuria. Proteinuria was measured by spot urine dipsticks (Roche) at 3 and 12 weeks of age. Congenic mice for the HIVAN1 locus were generated by 10 generations of backcrossing to FVB. The proximal and distal ends of the congenic intervals were delimited by markers D3Mit61 (5.1 Mb) and D3Mit69 (57 Mb), respectively. Successful introgression of this interval into the FVB genetic background was further confirmed by typing 82 informative loci across the genome in the tenth generation. For gene expression studies, mice were studied at 6-8 weeks of age. We profiled whole kidney in 40 nontransgenic mice and $20 \mathrm{HIV}-1$ transgenic mice equally distributed across HIVAN1 genotype and gender. To minimize secondary effects of glomerulosclerosis on gene expression, the HIV-1 transgenic mice of each HIVAN1 genotype were matched for age and degree of renal injury (range $9 \%-55 \%$ glomerulosclerosis). To study the HIVAN2 locus and the Nphs2 cis-eQTL, we compared 4 B6-chr13 $3^{\mathrm{A}}$ (all female) and $6 \mathrm{~B} 6$-chr01 $1^{\mathrm{A}}$ mice ( 3 male $/ 3$ female) to 10 wild-type $\mathrm{B} 6$ mice ( 5 male $/ 5$ female). Renal histology was scored independently by an investigator blinded to genetic background and other traits. Three traits related to tubulointerstitial disease (epithelial regeneration/degeneration, tubular casts and dilatation, and interstitial infiltrates) and 3 related to glomerular injury (glomerular sclerosis and podocyte hyperplasia and collapse) were scored using a semi-quantitative scale: 0 , no disease; $1,1 \%-25 \%$ of tissue showing abnormalities; $2,26 \%-50 \%$ of tissue showing abnormalities; 3 , $51 \%-75 \%$ of tissue showing abnormalities; and $4,>75 \%$ of tissue showing abnormalities. A global histologic score was calculated as an average of 6 renal injury scores. The protocol was approved by the IACUC at the Columbia University Medical Center.

Measurement of podocyte gene expression. We examined Nphs 2 expression in whole kidney because the gene is exclusively expressed in the glomerulus and demonstrated strong heritability from whole kidney measurements of inbred strains. Moreover, the acquisition of whole kidney tissue greatly minimizes the chance of producing secondary changes in gene expression 
compared with glomerular isolation procedures. Total RNA was extracted from mouse kidneys using TRIzol reagent (Invitrogen), followed by treatment with DNaseI and clean-up using the RNeasy kit (QIAGEN) according to the manufacturers' protocols. cDNA was generated with the OmniScript kit (Qiagen). Gene expression was quantitated in quadruplicate by quantitative PCR using SYBR Green mix on an IQ thermal cycler (Bio-Rad). Values were standardized to an internal control, a reference sample (a male FVB) included in each run, and $\beta$-actin was used as housekeeping control (using the Pfaffl algorithm; ref. 62). Primer sequences and PCR conditions are included in Supplemental Table 1 (supplemental material available online with this article; doi:10.1172/JCI37131).

Genotyping and analysis of linkage. The BC group had been previously genotyped using microsatellites across the mouse autosomes (45). The F2 cohort was genotyped using 103 informative SNPs across mouse autosomes (Kbiosciences). Pairwise and multipoint likelihood ratio statistics (LRSs) and the proportion of the variance of each trait explained by each trait locus were calculated using the QTX 20 program under an additive model (BC) and a free model (F2 cross) (63). Traits were adjusted for significant covariates by analysis of residuals. Lod scores were calculated by dividing the LRS by 4.6 , and traditional thresholds for significance in the $\mathrm{BC}$ and intercross were used (lod score $>3.3$ and $>4.3$, respectively) (64). In addition, we conducted 10,000 permutations of phenotypes on genotype to determine the empirical significance of the linkage findings. Significant linkage threshold was defined as the lod value that would be expected to occur by chance in 1 of 20 genome scans. Suggestive linkage was defined as the lod value that would be expected to occur at random once per genome scan. Confidence intervals were derived by bootstrap tests. The results of the analysis were also confirmed using the R/QTL package (http://www.rqtl. org/download/; ref. 65) and the Haley-Knott regression method. We performed a standard test of linkage versus pleiotropy to determine whether the coincident linkage peaks for HIVAN and the Nphs 2 transcript level were due to chance or attributable to pleiotropic effects of the same gene affecting both traits (47). In this analysis, we tested the hypothesis that 2 distinct genes that were closely located within the QTL locus influence each trait separately (linked QTL model) against the hypothesis that 1 gene in the interval influences both traits (pleiotropic QTL model). An LRS was computed for the linkage versus pleiotropy models, testing whether the best locations for the 2 QTL models were the same. A parametric bootstrap procedure was performed to obtain the null distribution. $P$ values less than 0.05 provided statistically significant evidence against the pleiotropic model. As a second approach for examining the possibility of chance overlap, we assumed complete independence between the nephropathy and Nphs2 expression traits and performed simulation studies in an F2 and a $\mathrm{BC}$ cohort of the same size as in our study. We first fixed the location of the HIVAN loci in each cross. We next performed 10,000 simulated genome scans containing 2 QTLs with the same effect size and confidence intervals as the Nphs 2 trans-eQTLs and randomly permutated the location of these eQTLs across the genome to determine how frequently confidence intervals demonstrate partial overlap (defined as $>10 \mathrm{cM}$ overlap) or complete overlap (best position within $5 \mathrm{cM}$ of each other). To test whether the data in congenic or consomic strains could be explained by multiple independent eQTLs within the substituted segments, we used the largest published mouse genome study (28) to estimate the density of trans-eQTLs across the genome and the a priori probabilities that a given transcript had a transregulator. The probability of detection of 5 independent genome-wide significant trans-eQTLs for 21 independent comparisons within the tested segment was derived from the binomial distribution.

Sequencing and 5' RACE and 3' RACE. The Nphs2 gene was sequenced in 19 inbred strains ( $2.5 \mathrm{~kb}$ upstream sequence upstream of the start codon, exons, and $3^{\prime}$ UTR), and sequence traces were aligned and analyzed using the Sequencher 4.7 program (Gene Codes Corp.). Polymorphism data are reported in Supplemental Tables 2-4 and also submitted to GenBank (accession numbers: FJ715751-FJ715776 and FJ717826-FJ717833). 5' RACE and 3' RACE experiments were performed according to the manufacturer's protocols (Ambion). TOPO TA cloning of the resulting PCR fragments into the pCRII vector (Invitrogen) with followed by sequencing confirmed identified variants.

Western blotting. Whole kidney lysates (100 $\mu$ g of total protein per well) were separated in $7.5 \%$ SDS-PAGE and transferred onto nitrocellulose membrane. Detection of proteins of interest was performed using Immobilon Western ECL detection kit (Millipore) and the following antibodies: polyclonal rabbit anti-podocin (Sigma-Aldrich), mouse monoclonal anti-GAPDH (R\&D Systems), and HRP-labeled sheep anti-mouse (Amersham Biosciences) or goat anti-rabbit (Jackson ImmunoResearch Laboratories Inc.) antibody.

Statistical and bioinformatics analysis. Statistical analyses were performed using SPSS version 15.0. Quantitative traits in the F2 group were compared using $t$ tests and ANOVA. Gene expression values among inbred strains were compared using a 2 -sided $t$ test. The generalized linear model procedure was used for analysis of gene expression in HIV-1 transgenic HIVAN1 congenic mice, adjusting for glomerulosclerosis. $P$ values $\leq 0.05$ were considered significant. Phylogenetic analyses of $N p h s 2$ sequences were performed using the MEGA 4.0 program (http://www.megasoftware.net/; ref. 66), using the unweighted pair group method with arithmetic mean (UPGMA) algorithm. In order to evaluate whether the high mutation rate in the Nphs 2 gene was due to positive selection, we estimated the ratio of non-synonymous $(\mathrm{Ka})$ to synonymous $(\mathrm{Ks})$ nucleotide substitution rates for each amino acid site within the Nphs 2 gene based on the analysis of homologous coding sequences from 10 different strains (67).

\section{Acknowledgments}

This work is supported by NIH grant 1PO1 DK56492-01. A.G. Gharavi is supported by the Columbia Center for Glomerular Diseases and the Emerald Foundation. This study was also supported by the histology core facility of Columbia Diabetes and Endocrinology Research Center (NIH grant P30-DK63608).

Received for publication September 30, 2008, and accepted in revised form March 4, 2009.

Address correspondence to: Ali G. Gharavi, Department of Medicine, Columbia University College of Physicians and Surgeons, 1150 St. Nicholas Ave., Russ Berrie Pavilion 302, New York, New York 10032, USA. Phone: (212) 851-5556; Fax: (212) 305-5520; E-mail:ag2239@columbia.edu.
1. Kestila, M., et al. 1998. Positionally cloned gene for a novel glomerular protein - nephrin - is mutated in congenital nephrotic syndrome. Mol. Cell. 1:575-582.

2. Kaplan, J.M., et al. 2000. Mutations in ACTN4, encoding alpha-actinin-4, cause familial focal segmental glomerulosclerosis. Nat. Genet. 24:251-256.

3. Boute, N., et al. 2000. NPHS2, encoding the glomerular protein podocin, is mutated in autosomal recessive steroid-resistant nephrotic syndrome. Nat. Genet. 24:349-354.

4. Kim, J.M., et al. 2003. CD2-associated protein haploinsufficiency is linked to glomerular disease susceptibility. Science. 300:1298-1300.

5. Winn, M.P., et al. 2005. A mutation in the TRPC6 cation channel causes familial focal segmental glomerulosclerosis. Science. 308:1801-1804.

6. Hinkes, B., et al. 2006. Positional cloning uncovers mutations in PLCE1 responsible for a nephrotic syndrome variant that may be reversible. Nat. Genet. 38:1397-1405.

7. D'Agati, V.D. 2008. Podocyte injury in focal segmental glomerulosclerosis: Lessons from animal models (a play in five acts). Kidney Int. 73:399-406.

8. Kao, W.H., et al. 2008. MYH9 is associated with nondiabetic end-stage renal disease in African Americans. Nat. Genet. 40:1185-1192. 
9. Kopp, J.B., et al. 2008. MYH9 is a major-effect risk gene for focal segmental glomerulosclerosis. Nat. Genet. 40:1175-1184

10. Freedman, B.I., Soucie, J.M., Stone, S.M., and Pegram, S. 1999. Familial clustering of end-stage renal disease in blacks with HIV-associated nephropathy. Am. J. Kidney Dis. 34:254-258.

11. Freedman, B.I., Soucie, J.M., and McClellan, W.M. 1997. Family history of end-stage renal disease among incident dialysis patients. J. Am. Soc. Nephrol. 8:1942-1945.

12. Kelley, M.J., Jawien, W., Ortel, T.L., and Korczak, J.F. 2000. Mutation of MYH9, encoding non-muscle myosin heavy chain A, in May-Hegglin anomaly. Nat. Genet. 26:106-108.

13. Seri, M., et al. 2000. Mutations in MYH9 result in the May-Hegglin anomaly, and Fechtner and Sebastian syndromes. The May-Heggllin/Fechtner Syndrome Consortium. Nat. Genet. 26:103-105.

14. Heath, K.E., et al. 2001. Nonmuscle myosin heavy chain IIA mutations define a spectrum of autosomal dominant macrothrombocytopenias: MayHegglin anomaly and Fechtner, Sebastian, Epstein, and Alport-like syndromes. Am. J. Hum. Genet. 69:1033-1045.

15. Ghiggeri, G.M., et al. 2003. Genetics, clinical and pathological features of glomerulonephritis associated with mutations of nonmuscle myosin IIA (Fechtner syndrome). Am. J. Kidney Dis. 41:95-104.

16. Arrondel, C., et al. 2002. Expression of the nonmuscle myosin heavy chain IIA in the human kidney and screening for MYH9 mutations in Epstein and Fechtner syndromes. J. Am. Soc. Nephrol. 13:65-74.

17. Deller, T., et al. 2003. Synaptopodin-deficient mice lack a spine apparatus and show deficits in synaptic plasticity. Proc. Natl. Acad. Sci. U. S. A. 100:10494-10499.

18. Putaala, H., Soininen, R., Kilpelainen, P., Wartiovaara, J., and Tryggvason, K. 2001. The murine nephrin gene is specifically expressed in kidney, brain and pancreas: inactivation of the gene leads to massive proteinuria and neonatal death. Hum. Mol. Genet. 10:1-8.

19. Roselli, S., et al. 2004. Early glomerular filtration defect and severe renal disease in podocin-deficient mice. Mol. Cell. Biol. 24:550-560.

20. Henderson, J.M., Al-Waheeb, S., Weins, A., Dandapani, S.V., and Pollak, M.R. 2008. Mice with altered alpha-actinin-4 expression have distinct morphologic patterns of glomerular disease. Kidney Int 73:741-750.

21. Huber, T.B., et al. 2006. Bigenic mouse models of focal segmental glomerulosclerosis involving pairwise interaction of CD2AP, Fyn, and synaptopodin. J. Clin. Invest. 116:1337-1345.

22. Koop, K., et al. 2003. Expression of podocyte-associated molecules in acquired human kidney diseases. J. Am. Soc. Nephrol. 14:2063-2071.

23. Zhang, S.Y., et al. 2004. In vivo expression of podocyte slit diaphragm-associated proteins in nephrotic patients with NPHS2 mutation. Kidney Int. 66:945-954.

24. Brem, R.B., Yvert, G., Clinton, R., and Kruglyak, L. 2002. Genetic dissection of transcriptional regulation in budding yeast. Science. 296:752-755.

25. Schadt, E.E., et al. 2003. Genetics of gene expression surveyed in maize, mouse and man. Nature. 422:297-302

26. Rockman, M.V., and Kruglyak, L. 2006. Genetics of global gene expression. Nat. Rev. Genet. 7:862-872. 27. Hubner, N., et al. 2005. Integrated transcriptional profiling and linkage analysis for identification of genes underlying disease. Nat. Genet. 37:243-253.

28. Wang, S., et al. 2006. Genetic and genomic analysis of a fat mass trait with complex inheritance reveals marked sex specificity. PLoS Genet. 2:e15.

29. Schadt, E.E., et al. 2005. An integrative genomics approach to infer causal associations between gene expression and disease. Nat. Genet. 37:710-717.

30. Chen, Y., et al. 2008. Variations in DNA elucidate molecular networks that cause disease. Nature. 452:429-435.

31. Wyatt, C.M., and Klotman, P.E. 2007. HIV-1 and HIV-associated nephropathy 25 years later. Clin. J. Am. Soc. Nephrol. 2(Suppl. 1):S20-S24.

32. D'Agati, V., and Appel, G.B. 1997. HIV infection and the kidney. J. Am. Soc. Nephrol. 8:138-152.

33. Winston, J.A., et al. 2001. Nephropathy and establishment of a renal reservoir of HIV type 1 during primary infection. N. Engl. J. Med. 344:1979-1984.

34. Wyatt, C.M., and Klotman, P.E. 2007. HIV-associated nephropathy in the era of antiretroviral therapy. Am. J. Med. 120:488-492.

35. Bruggeman, L.A., et al. 2000. Renal epithelium is a previously unrecognized site of HIV-1 infection. J. Am. Soc. Nephrol. 11:2079-2087.

36. Sunamoto, M., Husain, M., He, J.C., Schwartz, E.J., and Klotman, P.E. 2003. Critical role for Nef in HIV-1-induced podocyte dedifferentiation. Kidney Int. 64:1695-1701.

37. Husain, M., et al. 2002. HIV-1 Nef induces proliferation and anchorage-independent growth in podocytes. J. Am. Soc. Nephrol. 13:1806-1815.

38. Husain, M., D’Agati, V.D., He, J.C., Klotman, M.E., and Klotman, P.E. 2005. HIV-1 Nef induces dedifferentiation of podocytes in vivo: a characteristic feature of HIVAN. AIDS. 19:1975-1980.

39. He, J.C., et al. 2004. Nef stimulates proliferation of glomerular podocytes through activation of Srcdependent Stat3 and MAPK1,2 pathways. J. Clin. Invest. 114:643-651.

40. Lu, T.C., et al. 2008. HIV-1 Nef disrupts the podocyte actin cytoskeleton by interacting with diaphanous interacting protein. J. Biol. Chem. 283:8173-8182.

41. Ross, M.J., Martinka, S., D’Agati, V.D., and Bruggeman, L.A. 2005. NF-kappaB regulates Fas-mediated apoptosis in HIV-associated nephropathy. J. Am. Soc. Nephrol. 16:2403-2411.

42. Kiryluk, K., Martino, J., and Gharavi, A.G. 2007. Genetic susceptibility, HIV infection, and the kidney. Clin. J. Am. Soc. Nephrol. 2(Suppl. 1):S25-S35.

43. Winston, J.A., Klotman, M.E., and Klotman, P.E. 1999. HIV-associated nephropathy is a late, not early, manifestation of HIV-1 infection. Kidney Int. 55:1036-1040

44. Kopp, J.B., et al. 1992. Progressive glomerulosclerosis and enhanced renal accumulation of basement membrane components in mice transgenic for human immunodeficiency virus type 1 genes. Proc. Natl. Acad. Sci. U. S. A. 89:1577-1581.

45. Gharavi, A.G., et al. 2004. Mapping a locus for susceptibility to HIV-1-associated nephropathy to mouse chromosome 3. Proc. Natl. Acad. Sci. U. S. A 101:2488-2493

46. Zhong, J., et al. 2005. Expression of HIV-1 genes in podocytes alone can lead to the full spectrum of HIV-1-associated nephropathy. Kidney Int. 68:1048-1060.
47. Knott, S.A., and Haley, C.S. 2000. Multitrait least squares for quantitative trait loci detection. Genetics. 156:899-911.

48. Frazer, K.A., et al. 2007. A sequence-based variation map of 8.27 million SNPs in inbred mouse strains. Nature. 448:1050-1053

49. Belknap, J.K. 2003. Chromosome substitution strains: some quantitative considerations for genome scans and fine mapping. Mamm. Genome. 14:723-732.

50. Chan, K.T., et al. 2008. Accelerated development of collapsing glomerulopathy in mice congenic for the HIVAN1 locus. Kidney Int. 75:366-372.

51. Mhatre, A.N., et al. 2007. Generation and characterization of mice with Myh9 deficiency. Neuromolecular Med. 9:205-215.

52. Matsushita, T., et al. 2004. Targeted disruption of mouse ortholog of the human MYH9 responsible for macrothrombocytopenia with different organ involvement: hematological, nephrological, and otological studies of heterozygous KO mice. Biochem. Biophys. Res. Commun. 325:1163-1171.

53. Ratelade, J., et al. 2008. Maternal environment interacts with modifier genes to influence progression of nephrotic syndrome. J. Am. Soc. Nephrol. 19:1491-1499.

54. Takemoto, M., et al. 2006. Large-scale identification of genes implicated in kidney glomerulus development and function. EMBO J. 25:1160-1174

55. Rohr, C., et al. 2002. The LIM-homeodomain transcription factor Lmx1b plays a crucial role in podocytes. J. Clin. Invest. 109:1073-1082.

56. Yvert, G., et al. 2003. Trans-acting regulatory variation in Saccharomyces cerevisiae and the role of transcription factors. Nat. Genet. 35:57-64.

57. Park, C.C., et al. 2008. Fine mapping of regulatory loci for mammalian gene expression using radiation hybrids. Nat. Genet. 40:421-429.

58. Ronald, J., Brem, R.B., Whittle, J., and Kruglyak, L. 2005. Local regulatory variation in Saccharomyces cerevisiae. PLoS Genet. 1:e25.

59. GuhaThakurta, D., et al. 2006. Cis-regulatory variations: a study of SNPs around genes showing cis-linkage in segregating mouse populations. BMC Genomics. 7:235.

60. Doss, S., Schadt, E.E., Drake, T.A., and Lusis, A.J 2005. Cis-acting expression quantitative trait loci in mice. Genome Res. 15:681-691.

61. Fay, J.C., and Wu, C.I. 2001. The neutral theory in the genomic era. Curr. Opin. Genet. Dev. 11:642-646.

62. Pfaffl, M.W. 2001. A new mathematical model for relative quantification in real-time RT-PCR. Nucleic Acids Res. 29:e45.

63. Manly, K.F., Cudmore, R.H., Jr., and Meer, J.M. 2001. Map Manager QTX, cross-platform software for genetic mapping. Mamm. Genome. 12:930-932.

64. Lander, E., and Kruglyak, L. 1995. Genetic dissection of complex traits: guidelines for interpreting and reporting linkage results. Nat. Genet. 11:241-247.

65. Broman, K.W., Wu, H., Sen, S., and Churchill, G.A. 2003. R/qtl: QTL mapping in experimental crosses. Bioinformatics. 19:889-890.

66. Kumar, S., Tamura, K., and Nei, M. 2004. MEGA3: Integrated software for Molecular Evolutionary Genetics Analysis and sequence alignment. Brief Bioinform. 5:150-163.

67. Doron-Faigenboim, A., Stern, A., Mayrose, I., Bacharach, E., and Pupko, T. 2005. Selecton: a server for detecting evolutionary forces at a single amino-acid site. Bioinformatics. 21:2101-2103. 